Rafael García

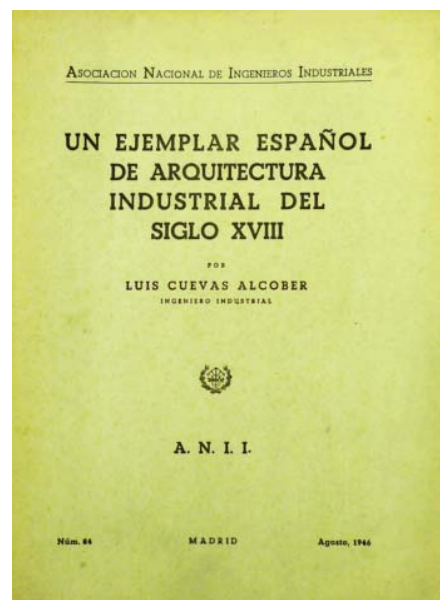

Luis Cuevas Alcober. Un ejemplar español de arquitectura industrial del siglo XVIII. Madrid, Asociación Nacional de Ingenieros Industriales, 1924.

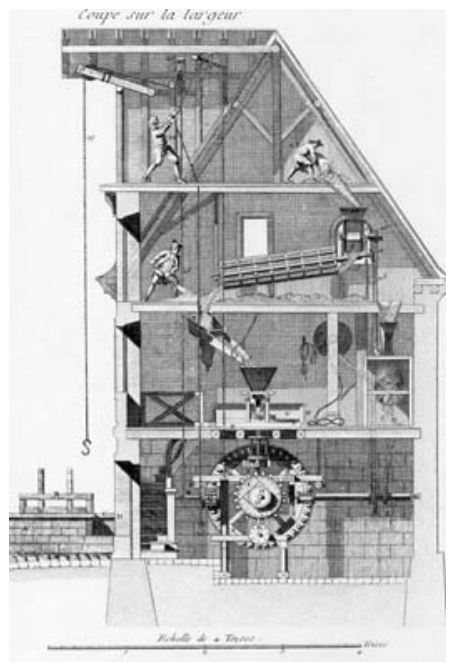

Molino hidráulico. Encyclopédie, Diderot.

\section{Arquitectura e industria Una exploración bibliográfica en la biblioteca de la Escuela de Arquitectura de la Universidad Politécnica}

Palabras clave: fábrica, exposición, bibliografia, patrimonio industrial, arquitectura industrial, ETSAM.

En este breve trabajo documental se recogen clasificados y ordenados los principales fondos bibliográficos existentes en la biblioteca de la Escuela Técnica Superior de Arquitectura (ETSAM) de la Universidad Politécnica de Madrid sobre el tema de la arquitectura y el patrimonio industrial. El motivo que dio lugar a esta búsqueda específica ha sido la exposición que con el título "La Fábrica: arquitectura industrial en los fondos de la Biblioteca ETSAM" se inauguró el 8 de abril de 2015. Su propósito es intentar que el trabajo de selección de fondos no se pierda tras el desmontaje de la exposición y que pueda estar disponible para estudiosos e interesados. Por su carácter se han excluido los fondos relativos a las obras públicas.

Dicha exposición forma parte de la contribución de esta escuela y su biblioteca a las actividades del programa especial 015 PI Patrimonio Industrial y Desarrollo en el cual participa como organizadora el Aula de Gestión e Intervención en el Patrimonio Arquitectónico e Industrial (Aula G+I PAI) de la Universidad Politécnica de Madrid. Esta exposición es la primera de una serie de tres sobre el mismo tema y a la que seguirán la que se inaugurará próximamente en el Colegio Oficial de Madrid con fondos de su biblioteca y una última en el rectorado de la Universidad Politécnica involucrando fondos de todas sus escuelas y a celebrar en el mes de noviembre de este año.

En la introducción realizada sobre la exposición de la ETSAM, se hacía una corta reseña acerca de algunos hitos históricos de la representación de lo industrial y su arquitectura que aquí reproducimos:

"La representación de la actividad industrial a través de sus mecanismos, dispositivos y construcciones es conocida al menos desde el Medioevo. Un ejemplo temprano es la miniatura del martirio de Saint Denis (1250), custodiado en la Bibliothèque Nationale de Paris, en la que un detalle describe con toda claridad la actividad de la obtención de harina en molinos flotantes sobre un curso de agua. Más tarde, ya en los comienzos del Renacimiento, el dibujo de máquinas y dispositivos técnicos encontró amplia difusión, teniendo a Mariano di Jacopo, conocido como Il Taccola, a un adelantado representante. Fue seguido de figuras como Francesco di Giorgio, Cantaglia o Leonardo, cuyos dibujos de ruedas y molinos se inspiraron en el anterior. Paralelamente, la obra de Giorgio Agrícola (George Baner) De Re Metálica (1556) - con dos ejemplares originales de la misma depositados en la bilblioteca de la Escuela de Minas de esta Universidad- daría lugar a la primera exposición sistemática grabada de la actividad metalúrgica y el laboreo de minas.

Todos ellos fueron jalones iniciales de una tradición que tendría más tarde un hito fundamental en la Ilustración a través de las láminas de la Encyclopédie de Diderot, ya en el XVIII. Sus cerca de 2900 láminas representando todas las industrias del momento y comenzadas a editarse en 1762 , superaron todas las expectativas al haberse iniciado con una promesa inicial de "solo"1000 grabados. Dicha tradición se siguió en las sucesivas enciclopedias y revistas "de la industria", aparecidas en el XIX 


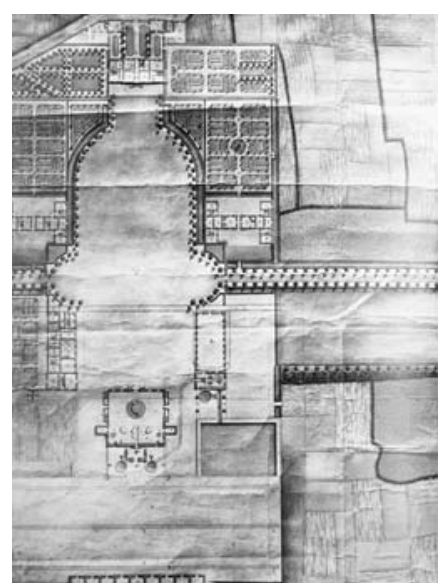

Fundición de Le Creusot, primer proyecto 1781. En Devillers $y$ Huet. Le Creusot: naissance et développement d'une ville industrielle 1782-1914, Seyssel, Champ Vallon, 1981.

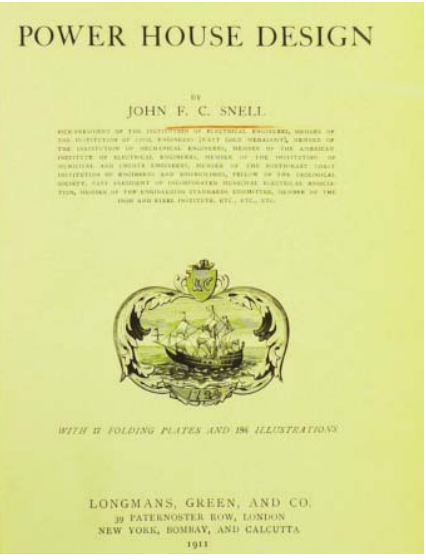

Johh F. C. Snell. Power house design. London, Longmans Green, 1911.

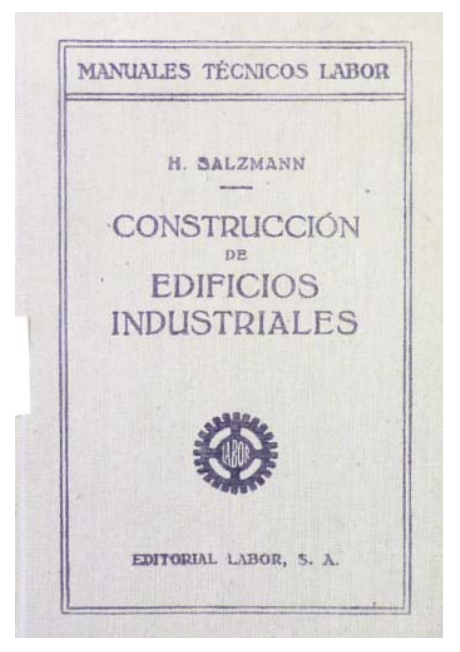

Heinrich Salzmann. Construcción de edificios industriales: almacenes, depósitos y fábricas. Barcelona, Labor, 1942. y editadas como expresiones genuinas de la idea de progreso, característica del siglo. De ellas son ejemplo los siguientes títulos conservados en esta misma biblioteca: La Gaceta industrial, económica y científica consagrada al fomento de la industria nacional (1865-1890), El museo de la industria: revista mensual de las artes industriales (1869-[1873]), Anales de la construcción y de la industria : periódico científico, artístico y comercial. (1876-1890), Arquitectura y construcción: bellas artes, decoración, industria, arte moderno (1897-1922) o la francesa Gazette du bâtiment : travaux publics, industrie, beaux arts (1860-1862).

Entretanto, una nueva institución, la fábrica, fue sustituyendo, ya desde comienzos de la revolución industrial, a las antiguas factorías y manufacturas. Con todo ello y con el nuevo impulso dado posteriormente por el mundo germánico y la Industriekultur a comienzos del XX, así como por las imágenes de silos y estructuras fabriles provenientes de América, la construcción industrial llegó a convertirse en centro de atención arquitectónico y motor de innovación y cambio. Sus repercusiones en la constitución de la Arquitectura Moderna han sido ampliamente reconocidas. Así pues, la fábrica, entendida no solo como tipo arquitectónico sino como metáfora integradora de la arquitectura y los procesos industriales, siguió encontrando un lugar propio en los medios impresos, al representar de forma directa el mundo surgido de la revolución industrial. Como tal, e incrementando su importancia a todo lo largo del siglo $\mathrm{XX}$ en las sucesivas revoluciones industriales, ha continuado apareciendo en múltiples publicaciones hasta nuestros dias."

Y a todo ello, y sin albergar pretensiones de exhaustividad, podriamos agregar aquí, con la sola excusa de que en este mismo número ya aparecen citados en uno de sus artículos (La ley del reloj de Eduardo Prieto), otros tratados como el Bellifortis de Konrad Kyeser (1405), el Texaurus Regis Franciae de Guido de Vigevano (1335) o el anónimo Liber Machinarum et Mecanica, "cuyas páginas recogen ejemplos de la 'ciencia ficción' del medievo" y, así mismo, el "liminar y excelente manual" Essai sur la composition des machines publicado en París en 1808 por los ingenieros españoles, José María Lanz y Agustín de Betancourt. Pero también debe tenerse en cuenta que estos últimos, como otros muchos que proliferaron posteriormente, nos llevan más al mundo de las máquinas, objetos mecánicos y dispositivos de guerra que al de lo industrial o sus aplicaciones más específicamente considerado. Es por ello que reiteramos la importancia y el gran salto que en la representación industrial se dio con la publicación de la Encyclopèdie, destacando sobre un pasado en que la industria solo en contadas ocasiones fue reflejada en imágenes. No obstante es preciso indicar que ni siquiera la Encyclopèdie de Diderot fue la iniciativa pionera, ya que en 1675 la Real Academia Francesa de Ciencias, fundada por Colbert, había recibido el encargo de realizar una "investigación y descripción científica de la industria francesa". Dichos trabajos al parecer se realizaron a escaso ritmo y en parte ralentizados por el perfeccionismo de René Antoine Ferchault de Réaumur (16831757), naturalista, metalúrgico y director de la Academia durante un tiempo. Sin embargo sí llegó a elaborar una serie de planchas para una no publicada Descripción de artes y oficios. Al anunciarse la publicación de la colección de láminas de Diderot, aún años antes de su edición efectiva, fueron muchas las críticas surgidas por diversas razones pero entre ellas estuvo en lugar destacado la de Réaumur. Su queja era que las láminas prometidas se basaban o eran incluso copias de las ya preparadas por su equipo. Las investigaciones de Georges Huard publicadas en 1952 en Reveu d'histoire des sciences et de leurs applications comparando ambas series ha mostrado lo razonable de la demanda de Réaumur al menos para las primeras editadas. Así pues y dejando aparte otras consideraciones, hablaríamos como mínimo de un "precedente" a la Encyclopèdie aunque en rigor no un precedente editado y a disposición del público, sino de un importante trabajo preparatorio que no vio la luz. 


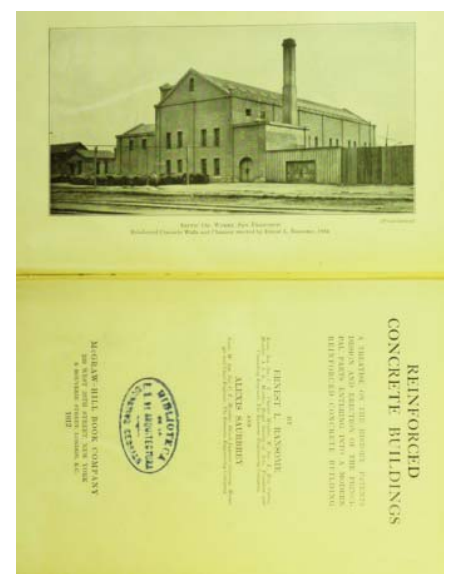

Ernest L. Ransome. Reinforced concrete buildings. New York, McGraw-Hill Book Company, 1912.

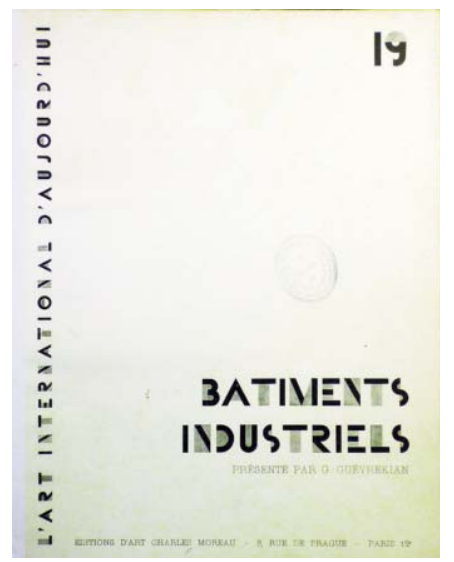

Gabriel Guévrékian. Batiments industriels. Paris, Éditions d'Art Charles Moreau, 19101940. (L'Art International d'Aujourd'hui).

\section{La selección}

Con la relación de títulos que consignamos a continuación intentamos que el trabajo de selección de fondos no se pierda tras el desmontaje de la exposición y que pueda estar disponible para estudiosos e interesados en estas cuestiones de una manera accesible. Tanto más cuanto que, como se indicaba en el texto acompañante, por "el carácter transversal y muchas veces no explícito de este material, su selección ha supuesto una búsqueda especial y nunca realizada anteriormente sobre los fondos de esta biblioteca". Todo ello ha dado lugar a unos materiales a veces heterogéneos en los que no solo están los libros monográficos, sean de autores, obras o temáticas, sino también otros como los catálogos de empresa, las enciclopedias o las recopilaciones de láminas, estas últimas además no necesariamente recogidas bajo un título general alusivo a lo industrial.

Como aspecto destacable referente al valor de antigüedad de lo expuesto incluimos aquí también la última parte del texto de la exposición: "En relación a titulos extranjeros de cierta antigüedad, entre los franceses son de destacar el texto de François Cointreax (1791), el Recueil de William y Farge (1873) o ya más moderna, la colección de láminas Batiments industriels de G. Gévrékian (1910-1940). También contiene abundantes láminas de arquitectura industrial la Encyclopédie de l'Architecture, editions Albert Morancé (sf). Entre los de lengua inglesa están la singular monografia sobre centrales térmicas, Power house design, de John F.C. Snell (1911), el tratado del angloamericano Ramsome, Reinforced concrete buildings (1912); la monografia sobre Albert Kahn de G. Nelson (1939) y el manual Planning Industrial structures de C. Dunham (1948). Italia aparece con un solo volumen, Gli edifici per le industrie de A. Melis (1953) y Alemania, la más representada en este aspecto, con la monografia sobre los nuevos edificios industriales de la Siemens, de H. Hertlein y H. Schmitz (1927), la monografia Ein Industriebau de E.G. Schmohl (1927), Russlend, Europa, Amerika de Mendelsohn (1929), el también monográfico Erich Mendelsohn de R. Mosse (1930) y el volumen de H. Maier-Leibnitz Der Industriebau (1932). La lámina del Matadero de Madrid, de Luis Bellido, corresponde a una exposición de 1911".

Por limitación de espacio, los títulos expuestos fueron solo una parte reducida del listado general aquí reflejado, por lo que en el mismo se resaltan los que han formado parte de la exposición. La elaboración inicial del listado, algo ampliada con nuevas búsquedas para esta publicación, fue realizada por Susana Feito por parte de la biblioteca con la colaboración de quien esto suscribe. Con todo, el trabajo no debe entenderse como una relación exhaustiva de títulos, entre otras cosas por su dificultad, ya que son muchas las obras no específicas -como por ejemplo los manuales generales de construcción- que pueden tener contenidos de carácter industrial, pero que no se han contemplado aquí al centrase la búsqueda en la literatura más directamente alusiva al tema. Por otra parte, dado que el objetivo ha sido principalmente el patrimonio industrial arquitectónico, se han excluido los fondos relativos a las obras públicas. En los listados se incluyen separadamente ediciones en diferentes idiomas de una misma obra.

Manuales, teoria, historia, rehabilitación.

Adam, Jürgen. Industrial buildings: a design manual. Basel, Birkhäuser, 2004.

Aguilar Civera, Inmaculada.Arquitecturas del Tram : la estación de Luceros : nueva plaza de la ciudad. Cátedra Demetrio Ribes UVEG-FG, Conselleria d'Infraestructures i Transport, 2010. 


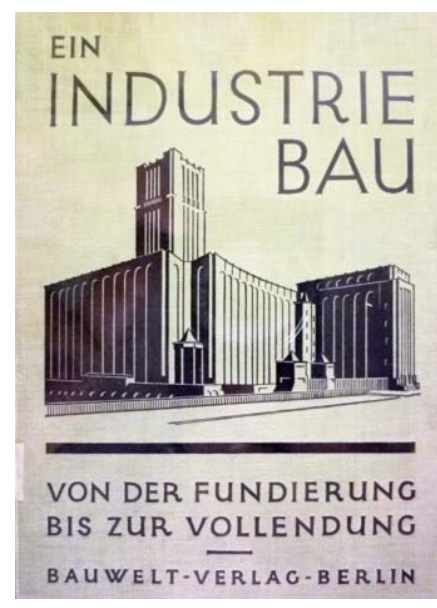

Eugen G. Schmohl. Ein industriebau, Berlin, Bauwelt-verlag, 1927.

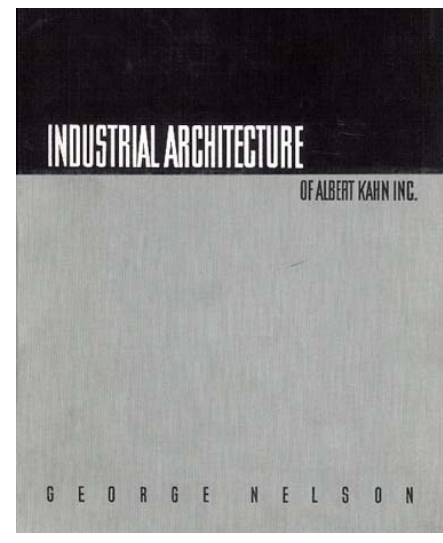

George Nelson. Industrial architecture of Albert Kahn, Inc. New York, Architectural Book Publishing, 1939
Aguilar Civera, Inmaculada. El patrimonio arquitectónico industrial. Cuadernos del Instituto Juan de Herrera de la Escuela de Arquitectura de Madrid, 1998.

Aguilar Civera, Inmaculada. La critica de la arquitectura y la ingeniería entre 1876 y 1890. M Cardereda, J.A. Rebolledo, E. M. Repullés, E. Saavedra y los Anales de la Construcción y de la Industria. Universidad de Valencia, Departamento de Historia del Arte, 1995.

Cardellach, Félix. Las formas artísticas en la arquitectura técnica: tratado de ingenieria estética. Barcelona, Agustín Bosch, 1916.

Catálogo. Architecture et industrie: passé et avenir d'un mariage de raison. Centre de Création Industrielle, 1983.

Cointeraux, François. Traité Sur La Construction Des Manufactures Et Des Maisons De Campagne, en Leçons de l'ècole gratuite de dessin et d'architecture de la ville de perpingan composées par Boher. Perpignan, Chez J. Alzine, 1791.

Darley, Gillian; García García, Rafael. La fábrica como arquitectura: facetas de la construcción industrial, Barcelona, Reverté, 2010.

Dunham, Clarence W. Planning industrial structures. New York etc., Mac Graw-Hill, 1948.

Marsh, Paul. The refurbishment of commercial and industrial buildings. London, Construction Press, 1983.

Ferrer Marsal, Juan. El faro de Alicante: una recuperación del patrimonio portuario. Cátedra Demetrio Ribes UVEG-FGV, 2008.

Melis, Armando y Vittorio Zignoli (notas técnicas). Gli edifici per le industrie: evoluzione dell'edificio industriale. Torino, S. Lattes \& C. editori, 1953.

Ransome, Ernest L. Reinforced concrete buildings. New York, McGraw-Hill Book Company, 1912.

Salzmann, Heinrich. Construcción de edificios industriales: (almacenes, depósitos y fábricas). Barcelona, Labor, 1942. (Manuales Técnicos Labor).

Sebastiá Talavera, Jordi. La belleza industrial: historia de la fábrica y su estética. Valencia, Fundación Bancaja, 2007

Stratton, Michael. Industrial buildings: conservation and regeneration. London, New York, E \& FN Spon, 2002.

\section{Arqueologia industrial}

Álvarez Areces, Miguel ángel. Arqueología industrial: el pasado por venir, Gijón, Cicees, 2007.

Borsi, Franco. Introduzione alla archeologia industriale. Roma, Officina Edizione, 1978.

Florido Castro, Amara M. Arqueología industrial en Las Palmas de Gran Canaria durante la Restauración (1869-1931). Cabildo de Gran Canaria, 1999.

Ibáñez López, Maite. Arqueología industrial en Bizkaia. Bilbao, Universidad de Deusto, 1988.

Martín Martínez, José. El ferrocarril minero de ojos-negros a Sagunt: arqueología industrial y patrimonio ferroviario. Universidad de Valencia, Facultad Geografia i Historia, 1995.

Trachana, Angelique. Arqueología industrial y restauración ambiental, Buenos Aires, Nobuko, 2008.

Trinder, Barrie. The Blackwell encyclopedia of industrial archaeology. Blackwell, 1992.

\section{Recopilaciones}

I Jornadas sobre la Protección y Revalorización del Patrimônio Industrial. San Sebastián, Gobierno Vasco, Departamento de Cultura, 1984.

Alonso del Val, Miguel A. Arquitectura industrial. Madrid, Munilla-Lería, 2003. 


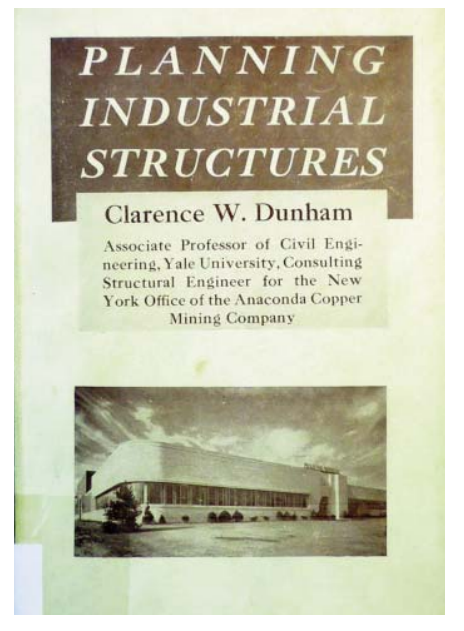

Clarence W. Dunham. Planning industrial structures. New York, Mac Graw-Hill, 1948.

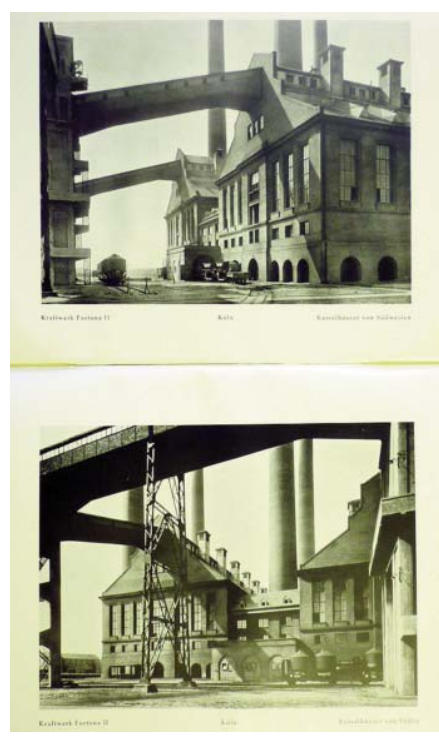

Hans Hertlein y Hermann Schmitz. Neue Industriebauten des Siemenskonzerns: Fabrik und Verwaltungsgebäude Wohlfahrtsanlagen. Berlin, Ernst Wasmuth, 1927.
Asensio Cerver, Francisco. Industrial buildings-Edificios industriales. Barcelona, Atrium, 1992.

Diderot, Denis, y D'Alambert. Recueil de planches sur les sciences, les arts libéraux, et les arts méchaniques, avec leur explication. Paris, Henri Veyrier, 1965. Repr. facs. de la edición de 1762. 24 v.

Diderot, Denis. Diderot Encyclopedia : the complete illustrations 1762-1777. Harry N. Abrams, 1978. 5 v.

Enclyclopèdie de l'architecture. Constructions modernes. Tome 1. Editions Albert Moranceé. 1930.

Guévrékian, Gabriel. Batiments industriels. Paris, Éditions d'Art Charles Moreau, 1910; 1940. (L'art international d'aujourd'hui).

Grube, Oswald W. Construcciones para la industria: selección internacional. Barcelona, Gustavo Gili, 1972. (Monografias de arquitectura).

Grube, Oswald W. Industrial buildings and factories. London, The architectural Press, 1971.

Henn, Walter. Bauten der industrie. Munchen, Georg D.W. Callwey, 1955. (Handbucher zur gestaltung von industrie- und verkehrsbauten)

Henn, Walter. Buildings for industry. London, Iliffe Books, 1965.

Henn, Walter. Atlas de la construction industrielle: projets et réalisations. Paris, Dunod, 1966.

Henn, Walter; Klaus Fangmeier; Ekko Flick y Gunther Lorf. Edificaciones industriales. Barcelona, Gustavo Gili, 1966.

Henn, Walter. Construction industrielle: examples internationaux. Paris, Dunod, 1967.

Maurer, H; T. Maurer y Richard P. Lohse. Neue industriebauten. Ravensburg, Otto Maier Verlag, 1954.

Phillips, Alan. Arquitectura industrial, Barcelona, Gustavo Gili, 1993.

Reid, Kenneth. Industrial buildings: the architectural record of a decade. New York, F. W. Dodge Corporation, 1951.

TICCIH. VIII Congreso internacional para la conservación del patrimonio industrial. Madrid, CEHOPU, 1992.

William y Farge (editores). Le recueil d'architecture. Paris, Librairie Générale de l'Architecture et des Travaux Publics Ducher, 1873-1896.

\section{Autores}

Aguilar, Inmaculada. Demetrio Ribes: arquitecto (1875-1921). Generalitat Valenciana, 2004.

Aguilar, Inmaculada. Demetri Ribes. Valencia, Eliseu Climent, 1980.

Garnier, Tony. Une cité industrielle: étude pour la construction des villes. Paris, Auguste Vincent, 1900; 1910.

Le Corbusier. Vers une architecture. Paris, Editions G. Crès, 1923.

Mendelsohn, Erich. Russlend, Europa, Amerika: ein architektonischer querschnitt. Berlin, Rudolf Mosse, 1929.

Hildebrandt, Grant. The architecture of Albert Kahn : Designing for industry. The MIT Press, 1974.

Nelson, George. Industrial architecture of Albert Kahn Inc. New York, Architectural Book Publishing Company, Inc, 1939.

Tchernikhov, Iakov. Iakov Tchernikhov. Documents et reproductions des archives de Aleksei e Dimitri Tchernikhov. Paris, Somogy Éditions d'Art, 1995.

Países, regiones, edificios y otros temas monográficos

AAVV. Industrial Heritage in Cities on Water. Número monográfico de: Aquapolis. Quarterly of International Centre Cities on Water. Año II, n. 4, December 1997.

Aguilar Civera, Inmaculada. La estación de ferrocarril, puerta de la ciudad. Generalitat Valenciana, Consellería de Cultura, Educació i Ciència, 1988.

Banham, Reyner. La Atlántida de hormigón: edificios industriales de los Estados Unidos y arquitectura moderna europea, 1900-1925, Madrid, Nerea, 1989. 


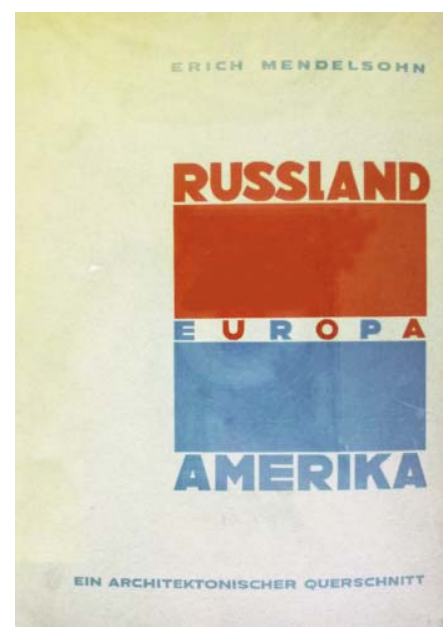

Erich Mendelsohn. Russland, Europa, Amerika: ein Architektonischer Querschnitt. Berlin, Rudolf Mosse, 1929.

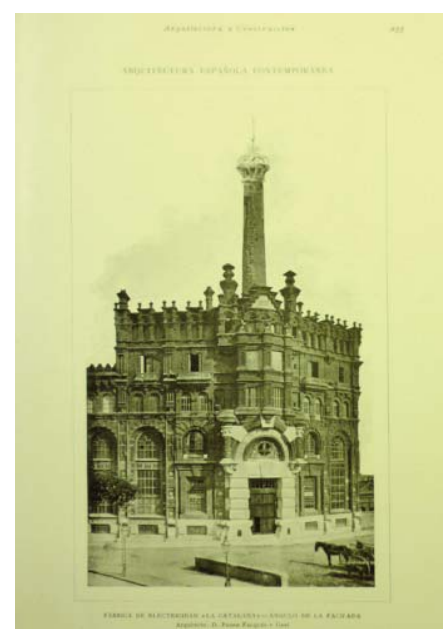

Pedro Falqués y Urpí. Fábrica de electricidad "La Catalana". Arquitectura y Construcción, 1901

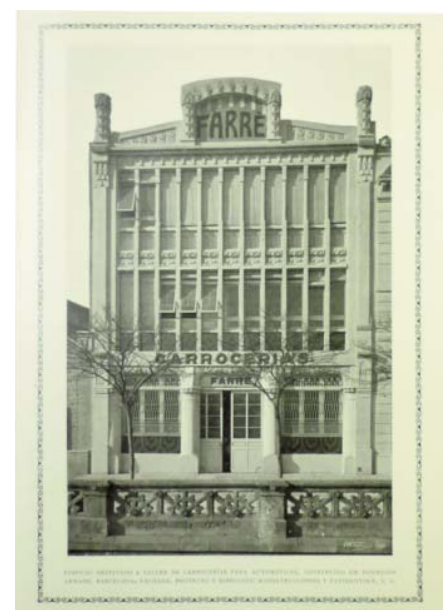

Carrocerías Farré (Barcelona). Arquitectura y Construcción, 1914.
Banham, Reyner. A concrete Atlantis: U. S. Industrial Building and European Modern Architecture 1900-1925, Cambridge, Massachusetts, MIT Press, 1989.

Bergeron, Louis; Maiullari-Pontois, María Teresa. Industry, architecture, and engineering. American ingenuity 1750-1950. Harry N. Abrams, 2000.

Bruttomesso, Rinio. Water and industrial heritage: the reuse of industrial and port structures in cities on water. Venice, Marsilio, 1999

Building Design Partnership. Power Station, Aldershot. S.1, 1967. (Report)

Devillers, Christian ; Huet, Bernard; Bergeron, Louis; Clément, Bernard y Sauvageot, Dominique. Le Creusot: naissance et développement d'une ville industrielle 1782-1914. Seyssel, Champ Vallon, 1981.

Ellsasser, Karl y Horst Ossenberg. Bauten der lebensmittel-industrie. Stuttgart, Julius Hoffmann, 19541.

Ferrier, Jacques. Usines. Paris, Le Moniteur, 1989.

Gray, W. S. Reinforced concrete water towers, bunkers, silos, and gantries. London, Concrete Publications, 1944.

Greiner, Lise. Lille 1830-1930: recherches sur l'architecture de 1830 à1930 dans la région lilloise effectuées de 1976 á 1978. Les Chateaux de l'industrie. Bruxelles, Archives d'Architecture Moderne, 1979.

Hertlein, Hans. Siemensbauten: neue Fabrik-und verwaltungsgebäude Wohlfahrtsanlagen des Siemenskonzern. Berlin, Ernst Wasmuth, 1900.

Hertlein, Hans y Hermann Schmitz. Neue Industriebauten des Siemenskonzerns: Fabrik und Verwaltungsgebäude Wohlfahrtsanlagen. Berlin, Ernst Wasmuth, 1927.

Linters, Adriaan. Architecture industrielle en Belgique. Bruxelles, Pierre Mardaga, 1986.

Mislin, Miron. The emergence of Berliner industrial buildings architecture: 1850-1910. Ausstellungsbau Räder, 2002.

Mislin, Miron. Industriearchitektur in Berlin: 1840-1910. Berlin, Wasmuth, 2002.

Schmohl, Eugen G. Ein industriebau: von de fundierung bis zur vollendung. Berlin, Bauwelt-verlag, 1927.

Snell, Johh F. C. Power house design. London etc, Longmans Green, 1911. (Longman's electrical engineering series).

Sommer, Degenhard. Architektur für die Arbeitswelt : neue bauten für industrie und gewerbe in Österreich. Basel, Birkhauser, 1995.

\section{España}

Aguilar Civera, Inmaculada. Las estaciones de ferrocarril en España: tipología y evolución: la Compañía de los Caminos de Hierro del Norte. Ed. del autor, 1986.

Aguilar Civera, Inmaculada. Las estaciones ferroviarias de Madrid: su arquitectura e incidencia en el desarrollo de la ciudad. Colegio Oficial de Arquitectos de Madrid, 1980.

Altos Hornos de Vizcaya. Monografia de la Sociedad Altos Hornos de Vizcaya: año 1909. Barcelona, Thomas, 1909

Biel Ibañez, María Pilar; Gerardo J. Cueto Alonso. 100 elementos del patrimonio industrial en España. Gijón, TICCIH España, 2011.

Biel Ibañez, María Pilar. Zaragoza y la industrialización : la arquitectura insdustrial en la capital aragonesa entre 1875-1936. Gobierno de Aragón, 2004.

Biel Ibáñez, María Pilar. Patrimonio industrial en la provincia de Zaragoza: Cinco villas. Institución Fernando el Católico, 2003.

Carrera de la Red, Miguel Ángel. La arquitectura de las fábricas de harina de la provincia de Valladolid. Tesis, 1987. $2 \mathrm{v}$.

Corredor-Matheos, José. Arquitectura industrial en Cataluña: del 1732 al 1929. Caja de Cataluña, 1984.

Cuevas Alcober, Luis. Un ejemplar español de arquitectura industrial del siglo XVIII. Madrid, Asociación Nacional de Ingenieros Industriales, 1946.

Díaz Díaz, Rafael; Diego Peris Sánchez. Arquitectura para la industria en Castilla-La Mancha. Albacete, Servicio de Publicaciones de la Junta de 


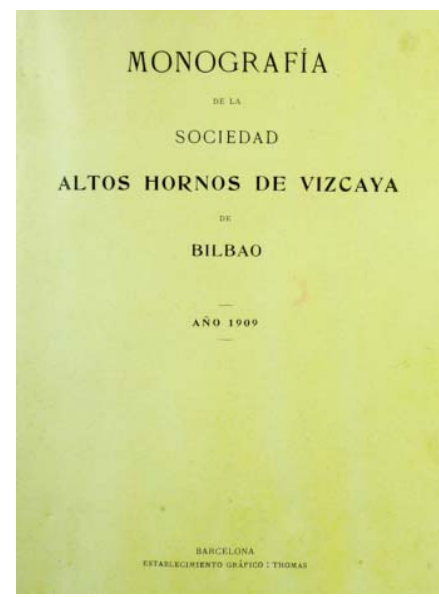

Monografia de la sociedad Altos Hornos de Vizcaya. 1909
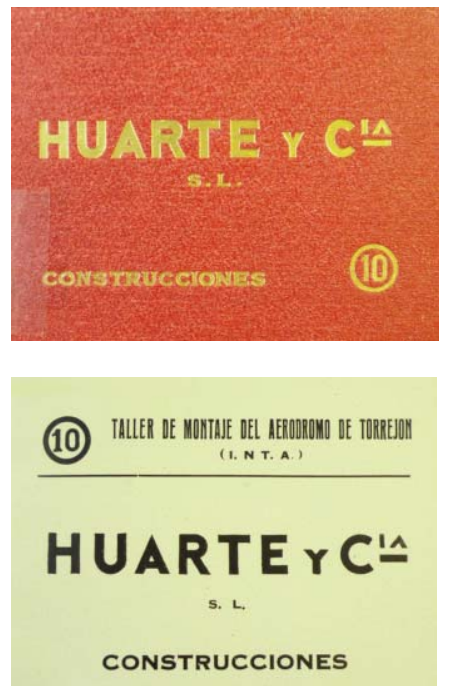

Huarte y Cía. Cuaderno n.10. Taller de montaje en el INTA, Torrejón.
Comunidades de Castilla-La Mancha, 1995. (Patrimonio histórico de Castilla-La Mancha).

D' Ors, Mauricio. Patrimonio industrial de Andalucia: portfolio fotográfico. Sevilla, Consejería de Obras Públicas y Transporte, 2006.

Hercé, José A. Apuntes sobre arquitectura industrial y ferroviaria en CastillaLa Mancha 1850-1936. Colegio Oficial de Arquitectos de Castilla-La Mancha, 1998.

Huarte y Compañia. Puerto de pasajes, estación de Santander. Madrid, Tipografia Artística, 1950. (Construcciones) .

Huarte y Compañia. Taller de montaje del aeródromo de Torrejón (INTA). Madrid, Tipografia Artística, 1950. (Construcciones)

Instituto del Patrimonio Cultural. Carta del Bierzo para la conservación del patrimonio industrial minero. Madrid, Ministerio de Cultura, 2009

Layuno, Ángeles (dir.). Minas de plata de Hiendelaencina. Territorio, Patrimonio y Paisaje. Universidad de Alcalá de Henares, 2014

Lucas, Antonio de; Diego Peris Sánchez; Angel Alcalde. La fábrica de armas de Toledo. The Toledo weapons factory. Ciudad Real, Universidad de Castilla-La Mancha, 2001.

Marqués Martín, Isabel. Segovia : inventario del patrimonio histórico industrial de la provincia de Segovia. Consejería de Cultura y Turismo, Junta de Castilla y León, 2008.

Martín Martínez, José. El ferrocarril minero de ojos-negros a Sagunt: arqueología industrial y patrimonio ferroviario. Universitat Valencia. Facultat Geografia i Historia, 1995.

Martín Martínez, José (editor). Urbanismo y arquitectura industrial en Puerto de Sagunto (1907-1936). Caja de Ahorros de Sagunto, 1990.

Navascués Palacio, Pedro. Arquitectura e ingeniería del hierro en España, (1814-1936). Madrid, El Viso, 2007.

Otamendi. Miguel. El ferrocarril metropolitano Alfonso XIII de Madrid. Madrid, Compañía Metropolitano Alfonso XIII, 1930.

Pardo Abad, Carlos J. Vaciado industrial y Nuevo paisaje urbano en Madrid: antiguas fábricas y renovación de la ciudad. Madrid, La librería, 2004.

Peris Sánchez, Diego. Paisajes industriales de Castilla-La Mancha. Bubok Publishing, 2013.

Peris Sánchez, Diego; Rafael Elvira Gutiérrez; Antonio de Lucas. El edificio Sabatini en la Fábrica de Armas de Toledo. Cuenca, Universidad de Castilla-La Mancha, 2001.

Peris Sánchez, Diego; Antonio de Lucas; Pedro Aranguez Ruiz. La fábrica de armas de Toledo. Ciudad Real, Universidad de Castilla-La Mancha, 1999.

Peris Sánchez, Diego. Dos siglos en construcción: de fábrica de armas a campus universitario. Grupo San José, 2003.

Rabanal Yus, Aurora. Las reales fundiciones españolas del siglo XVIII. Madrid, Estado Mayor del Ejército, Servicio de Publicaciones, 1990. (Colección Marte)

Rabanal Yus, Aurora. La Real Fábrica de Tejidos de Algodón de Ávila. Madrid, S.N., 1989.

Rabanal Yus, Aurora; Miguel Parrilla Nieto. Las Reales Fábricas de municiones de Eugui y Orbaiceta en Navarra. Pamplona, Gobierno de Navarra, Departamento de Educación y Cultura, 1987.

Rabanal Yus, Aurora. Arquitectura industrial del siglo XVIII en España: las reales fundiciones. Madrid, A. Rabanal Yus, 1986.

Rabanal Yus, Aurora; Instituto de Estudios Madrileños. En torno a la introducción y localización de las Reales Fábricas en el Madrid del siglo XVIII. Madrid, S.N., 1984. .

Reyes Mesa, José Miguel; Miguel Giménez Yanguas. Miradas desde el ferrocarril del azúcar: paisaje y patrimonio industrial en la Vega de Granada. Granada, Axares, 2014.

Sánchez de León Robles, Maria Victoria. Ávila : inventario del patrimonio histórico industrial de la provincia de Ávila. Consejería de Cultura y Turismo, Junta de Castilla y León, 2008.

Sobrino Simal, Julián. Arquitectura industrial en España, 1830 - 1990. Madrid, Cátedra, 1996. (Cuadernos Arte Cátedra) 


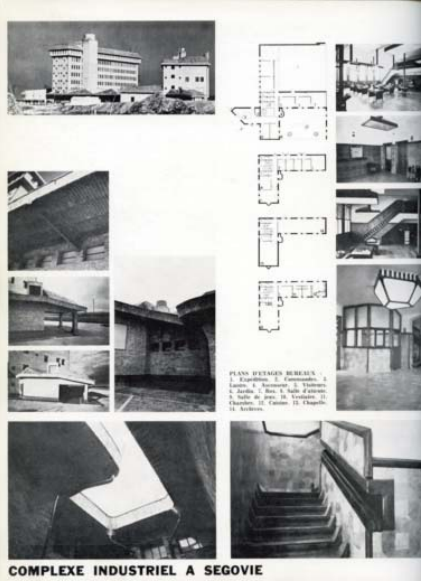

Inza, Curro. Complexe industriel a Segovia. L'Architecture d'Aujourd'hui, $n$. 149, 1970.

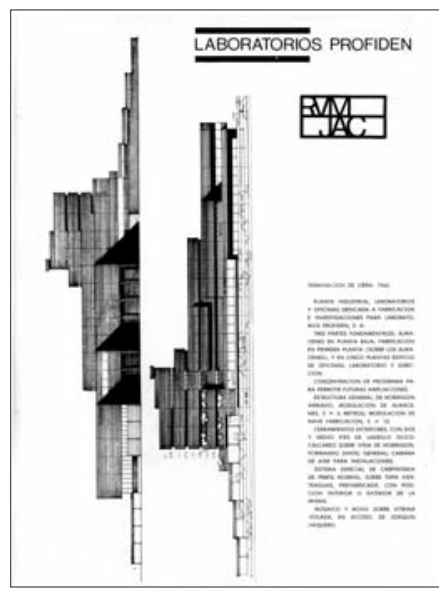

Corrales y Molezún.

Laboratórios profidén Madrid. Arquitectura, n. 94, 1966.

Luis Bellido. Matadero de Madrid. Fragmento de lámina para exposición de 1911.
Sobrino Simal, Julián. Arquitectura industrial en España: (1830-1990). Madrid, Banco de Crédito Industrial, 1989

Sobrino Simal, Julián. Arquitectura de la industria en Andalucía. Sevilla, Instituto de Fomento de Andalucía, 1998.

Tielve garcia, Natalia. Arquitectura moderna en la central de Soto de Ribera: la obra de Ignacio Álvarez Castelao y Carlos Fernández Casado. Gijón, CICEES, 2009.

Yravedra Soriano, María José. La arquitectura del vino en tres zonas españolas: Jerez, La Rioja y la Región del Cava. Tesis, 2002.

\section{Revistas}

(solo artículos y obras preseleccionados para exposición)

\section{Arquitectura y Construcción}

- Falqués y Urpí, Pedro. "Fábrica de electricidad La Catalana". 1901.

- Urioste y Velada, José. "El Nuevo Laboratorio Municipal, Madrid". 1903.

- "Fábrica De Cementos y Cales Hidráulicas De Los Sres. M.C.

Butsems y Fradera”. 1903.

- "Compañía General De Asfalto y Porland 'Asland”. 1905.

- Anasagasti, Teodoro de. "El Arte en Las Construcciones Industriales". 1914.

- "Edificio destinado a taller de carrocerias para automóviles, construido en hormigón armado: Carrocerias Farré (Barcelona)". 1914.

- "Central eléctrica de San Adrián, Barcelona. Central térmica y estación transformadora". 1914.

- Salvador, Amós. "Fábrica De Perfumería Gal, Madrid". 1917.

Informes de la Construcción

- Cano Lasso, Julio. "Manufacturas metálicas madrileñas". N. 57, 1954.

Arquitectura

- Corrales y Molezún. "Laboratórios Profidén Madrid". N.94, 1966.

- Peña Ganchegui, Luis. "Fábrica de calzado en Burgos". N.95, 1966. L'Architecture d'Aujourd'hui

-Inza, Curro. "Fábrica de chorizos en Segovia". N. 149, 1970.

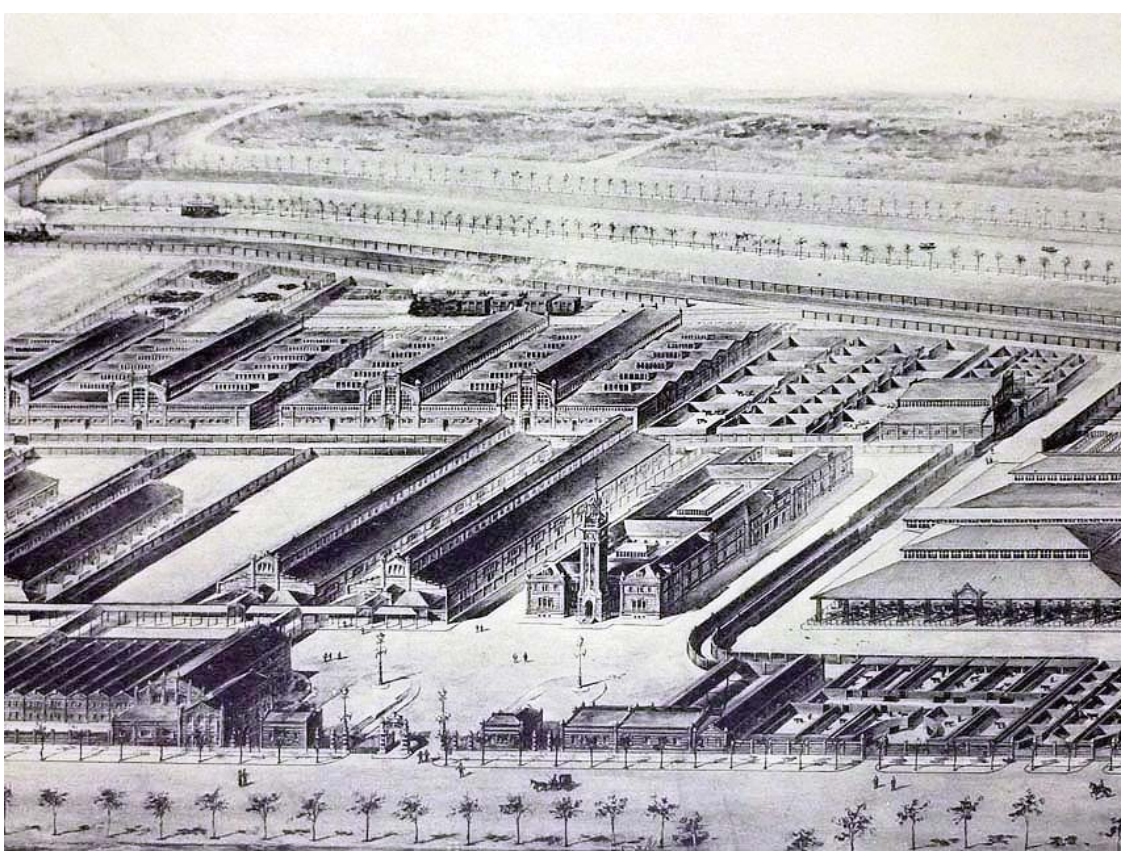

